\title{
Absorption of Femtosecond Laser Pulses in High-Density Plasma
}

\author{
R. Fedosejevs, ${ }^{(a)}$ R. Ottmann, and R. Sigel \\ Max-Planck-Institut für Quantenoptik, D-8046 Garching, Federal Republic of Germany \\ G. Kühnle, S. Szatmari, ${ }^{(b)}$ and F. P. Schäfer \\ Max-Planck-Institut für Biophysikalische Chemie, D-3400 Göttingen, \\ Federal Republic of Germany \\ (Received 26 June 1989)
}

\begin{abstract}
The absorption of 250 -fs $\mathrm{KrF}$ laser pulses incident on solid targets of aluminum and gold has been measured as a function of polarization and angle of incidence for the intensity range of $10^{14}-2.5 \times 10^{15}$ $\mathrm{W} \mathrm{cm}^{-2}$. Maximum absorption of over $60 \%$ occurs for $p$-polarized radiation at angles of incidence in the range of $48^{\circ}-57^{\circ}$. The measured results are in agreement with absorption on a steep density gradient.

PACS numbers: $52.50 . \mathrm{Jm}, 52.40 . \mathrm{Db}$
\end{abstract}

With the recent advent of high-intensity femtosecond lasers the study of laser-plasma interaction at high densities and high intensities on a subpicosecond time scale has become a vigorously studied topic. ${ }^{1-11}$ In this context the absorption and reflection of laser light by the highly conducting plasma formed at the target surface is of considerable interest. It is well known that for nanosecond and picosecond laser pulses the interaction is extremely complex because laser-light-induced instabilities have sufficient time to grow in an expanding plasma with extended density gradients. For femtosecond pulses the plasma has very little time to expand or for the instabilities to grow and one expects here an exceedingly simple situation: The absorption and reflection of the laser light should obey the laws of linear metal optics. In the investigation described in this Letter we have attempted to verify this hypothesis.

A laser pulse of infinitesimally short duration irradiating a solid target would interact with a steplike density profile. In the framework of linear optics reflection is given by the Fresnel-Drude formulas ${ }^{12}$ which predict a characteristic dependence of reflectivity versus angle of incidence, $\theta_{\text {inc }}$, and polarization. Maximum absorption occurs for $p$-polarized light at an optimum coupling angle, $\theta_{m}$. If the laser-pulse duration is finite the pulse interacts with an expanding electron-density profile. The angular variation of absorption still persists in a similar form, but the optimum angle decreases as the density scale length increases. The collisionless absorption of $p$ polarized radiation in a plasma with a finite density scale length is called resonance absorption. It was first observed, in a plasma produced by nanosecond pulses, by Manes et al. $;^{13}$ a large number of investigations followed. With the new interest in fs laser-plasma interactions it became clearly important to verify the angular dependence of laser-light absorption and its implications for the density scale length on this new ultrafast time scale. Recent fs experiments either have been performed only at a single angle of incidence ${ }^{1,2}$ or have shown a maximum at an angle of $0^{\circ}$ in contradiction with expectations. $^{3}$
The measurements presented in this Letter clearly demonstrate for the first time the characteristic angular dependence of laser-light absorption expected for the extremely steep density gradients of plasmas produced by femtosecond pulses. Furthermore, the measurements are accurate enough to show the difference between the angular dependence expected for a steplike density profile (as described by Fresnel's formulas) and that expected for the steep, but finite density gradient of a plasma expanding on a fs time scale. Results are presented for low- and high-atomic-number target materials using $\lambda=248 \mathrm{~nm} \mathrm{KrF}$ laser pulses which, due to their short wavelength, are of great interest for applications involving the creation and heating of very-high-density plasmas. We find quantitative agreement between the timeintegrated reflectivity data and calculations based on solving the wave equation for representative stationary plasma profiles.

The present experiments were conducted using a prism-compressed $150-\mathrm{fs} \mathrm{KrF}$ laser system ${ }^{14,15}$ with a wavelength of $\lambda_{0}=248 \mathrm{~nm}$ and pulse energies of up to 20 $\mathrm{mJ}$ on target. The $45 \times 24-\mathrm{mm}$ cross-section beam was focused by means of a $45-\mathrm{cm}$ focal-length $\mathrm{LiF}$ lens onto aluminum or gold targets in vacuum. The total optical system was designed to minimize the pulse-front distortion on target from the use of refractive optics. ${ }^{15,16}$ Measurements of the residual wave-front curvature gave a calculated pulse duration on target of $250 \pm 30 \mathrm{fs}$. The focal spot size was determined by transmission measurements through 50- and 30- $\mu$ m-diam pinholes giving an effective focal spot size of $56-\mu \mathrm{m}$ diameter. The polarization of the beam was measured in the target chamber, giving $93 \%$ of the total intensity in the desired polarization direction (either $s$ or $p$ polarized). This polarization mix has been used in all calculations of reflectivity when compared with the data.

The targets used for the present experiments were fresh films of aluminum and gold deposited on clean microscope slides. The thicknesses of the films were 0.8 and $1.3 \mu \mathrm{m}$ and the measured reflectivities at normal incidence for low-intensity $248-\mathrm{nm}$ light were $80 \%$ and 
$29 \%$, respectively, for aluminum and gold. The thickness is much greater than the expected interaction depth, $\sim 100 \mathrm{~nm}$, and thus the targets can be considered to be thick.

The total reflectivity was measured using an Ulbricht sphere for the diffuse part of the scattered light, a Gentec calorimeter for the specularly reflected component, and cross-calibrated photodiodes for the incident and backscattered energies. The Ulbricht-sphere coating material was tested using femtosecond uv laser pulses for linearity and stability within the operating intensity range. It was found that the direct specularly reflected component would be intense enough to give a nonlinear response in the integrating sphere and thus this part of the signal was measured directly with a calorimeter. It was covered with a calibrated LiF diffuser to block the expanding plasma and x-ray energy. All other energy detectors were cross calibrated against the Gentec calorimeter covered with the LiF diffuser. Typically, three or more shots were taken for each parameter condition. The shot-to-shot reproducibility was extremely good with a measured standard deviation over a number of shots of $\pm 4 \%$.

The measurements were carried out for two irradiation intensities of $I_{L}=10^{14}$ and $2.5 \times 10^{15} \mathrm{~W} \mathrm{~cm}^{-2}$ which corresponded to turning the final $\mathrm{KrF}$ amplifier module off or on, respectively. The prepulse intensity on target (for a duration of $\sim 7$ ns prior to the main pulse) was measured to be $6 \times 10^{5}$ and $1.4 \times 10^{8} \mathrm{~W} \mathrm{~cm}^{-2}$, respectively, for these two intensity ranges. The measured threshold for formation of preplasma on an aluminum target surface was $\sim 10^{8} \mathrm{~W} \mathrm{~cm}^{-2}$, and thus with the final amplifier turned off, the prepulse is well below the threshold for forming a preplasma. With the final amplifier turned on, a small amount of preplasma is expected on the target surface.

The experimentally measured total reflectivities as a function of angle of incidence for the two target materials and two intensity conditions are shown in Fig. 1. The low-intensity $s$-polarized data were obtained at a slightly lower intensity of $5 \times 10^{13} \mathrm{~W} \mathrm{~cm}^{-2}$ but we will primarily be concerned with the $p$-polarized data which were taken at $I_{L}=10^{14} \mathrm{~W} \mathrm{~cm}^{-2}$. In all cases the reflectivity for $s$ polarized radiation increases for larger angles of incidence. The reflectivity for $p$ polarization decreases with $\theta_{\text {inc }}$ up to an angle of minimum reflectivity, $\theta_{m}$, and then increases for larger angles. Note that for angles up to the angle of minimum reflectivity, the absorbed laser intensity remained roughly constant for the $p$-polarized data, because the decrease in reflectivity compensated for the increase in projected area of the laser spot. From a fit to the experimental data, we determined the angle for maximum absorption as $(54 \pm 3)^{\circ}$ and $(57 \pm 3)^{\circ}$ for aluminum and gold targets for $I_{L}=10^{14} \mathrm{~W} \mathrm{~cm}^{-2}$ and $(48 \pm 3)^{\circ}$ and $(50 \pm 3)^{\circ}$ for $I_{L}=2.5 \times 10^{15} \mathrm{~W} \mathrm{~cm}^{-2}$, respectively. It may be noted that the results depend only weakly on the target material and laser intensity.

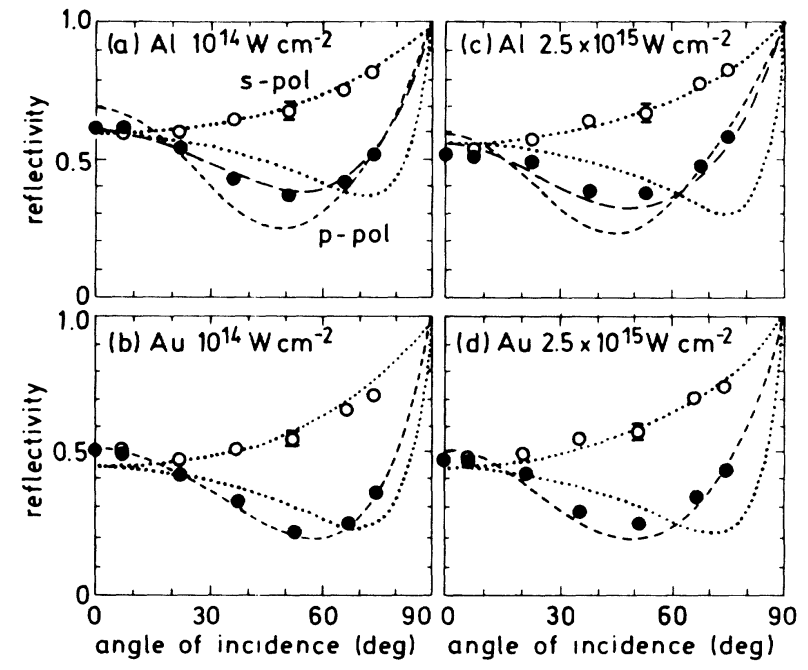

FIG. 1. Experimental measurements of total reflectivity as a function of the angle of incidence for $s$-polarized (open circles) and $p$-polarized (closed circles) radiation. The dotted curves are theoretical calculations for step density profiles and the dashed curves are numerical calculations for exponential (short dashed) and linear (long dashed) electron-density profiles: (a) aluminum, $I_{L}=10^{14} \mathrm{~W} \mathrm{~cm}^{-2}$; (b) gold, $I_{L}=10^{14} \mathrm{~W} \mathrm{~cm}^{-2}$; (c) aluminum, $I_{L}=2.5 \times 10^{15} \mathrm{~W} \mathrm{~cm}^{-2}$; and (d) gold, $I_{L}=2.5 \times 10^{15}$ $\mathrm{W} \mathrm{cm}^{-2}$

The use of the Ulbricht sphere made it possible to measure the diffuse part of the reflected light separately. It was always a small fraction of the total reflected signal, typically on the order of $(2-8) \%$ at the lower intensity and (4-14)\% of the incident radiation for the higher-intensity case. For all non-normal angles of incidence, $\theta_{\text {inc }} \geq 7.5^{\circ}$, no backscatter radiation was recorded under any of the conditions studied here. The absence of backscatter instabilities and the excellent reproducibility of the measurements suggest that even for the higher irradiation intensity the prepulse problem is under much better control than in previous experiments. ${ }^{3}$

A striking feature of the observations is their insensitivity to the target material and laser intensity. Measurements of absorption at lower intensities, using identical techniques, ${ }^{17}$ show clearly that the laser light is interacting with atoms of the bulk target material as opposed to a surface layer of some contaminant. The insensitivity to target material is expected and can be most readily shown by a comparison to Fresnel's formulas for a steplike density profile. From the Drude model of the plasma $^{12}$ the expected index of refraction, $n_{r}$, is given by $n_{r}^{2}=1-n_{e}^{*} /\left(1+i v^{*}\right)$, where $n_{e}^{*}=n_{e} / n_{c}$ is the normalized electron density, $n_{c}=m_{e} \omega^{2} / 4 \pi e^{2}$ is the critical density, $v^{*}=v / \omega$ is the electron collision frequency normalized to the laser frequency $\omega$, and $n_{e}$ and $m_{e}$ are the electron density and mass, respectively. According to the well-known Fresnel-Drude formulas ${ }^{12}$ the reflectivity of such a plasma is given by only two parameters, namely, the electron density and collision frequency, which, through the degree of ionization, are directly dependent 
on the temperature. The temperature was determined from the nonlinear electron heat wave propagating into the solid density. ${ }^{17,18}$ The heat conductivity and Coulomb logarithm were taken from Spitzer. ${ }^{19}$ The estimated electron temperatures, $T_{e}$, at $10^{14}$ and $2.5 \times 10^{15}$ $\mathrm{W} \mathrm{cm}{ }^{-2}$ are 57 and $310 \mathrm{eV}$, respectively, for the aluminum target and $220 \mathrm{eV}$ for the gold target at $2.5 \times 10^{15}$ $\mathrm{W} \mathrm{cm}{ }^{-2}$. The ionization states were calculated at solid density from a Thomas-Fermi model, ${ }^{20}$ giving values of $Z_{\text {eff }}$ of 6 and 11 for the two aluminum cases and 22 for gold at the higher intensity. For the gold target at $I_{L}=10^{14} \mathrm{~W} \mathrm{~cm}^{-2}$ the Coulomb logarithm is of the order of zero and the simple formula for thermal conductivity in the plasma is no longer applicable. The maximum temperature is expected to be less than that of aluminum and thus $Z_{\text {eff }}$ is estimated to be of the order of or less than 11 .

Reflectivity curves calculated from the Fresnel formulas are shown in Fig. 1. They were calculated for electron collision frequencies $v^{*}$ determined by a leastsquares fit to the experimental data using the estimated degree of ionization given above. It is seen from Fig. 1 that the calculated curves are quite consistent with the experimental measurements except for the location of the minimum in the $p$-polarized data (see discussion below). For aluminum at $10^{14} \mathrm{~W} \mathrm{~cm}^{-2}$ the observed reflectivity agrees very well with expectations (the collision frequency determined from the fit agrees within $10 \%$ with the value expected from the estimated temperature). At $2.5 \times 10^{15} \mathrm{~W} \mathrm{~cm}^{-2}$ one expects from the temperature estimates larger reflectivities than observed, namely, 0.72 and 0.65 for aluminum and gold at normal incidence, respectively. This discrepancy seems to be the only effect of the prepulse which we could identify in the experiments. The enhanced absorption is believed to occur in the leading edge of the main pulse as it "burns" through an underdense preformed plasma. As will become evident from the angular dependence of the data, the main part of the pulse interacts with the steep gradient close to the surface of the target.

The Fresnel formulas, valid for a sharp discontinuity, are not suitable to describe the angular dependence of the absorption data. This is obvious from Fig. 1 where the angle for minimum reflection of the calculated curves is on the order of $(70-74)^{\circ}$ which is much larger than the experimentally measured value. This discrepancy remains even if the collision frequencies or ionization levels are varied up to a factor of 2 times from their estimated values. This angular shift indicates a finite density gradient due to the expansion of the plasma. Unfortunately analytical expressions ${ }^{21}$ relating $\theta_{m}$ to the gradient length $L$ exist only in the limit $L>\lambda$ [where $\sin \theta_{m}=0.65\left(2 \pi L^{*}\right)^{-1 / 3}$ ]. Because the gradient length is much less than a wavelength in this experiment it was necessary to perform a numerical solution of the wave equation in the transition range.

Numerical results are shown in Fig. 2. It is found that

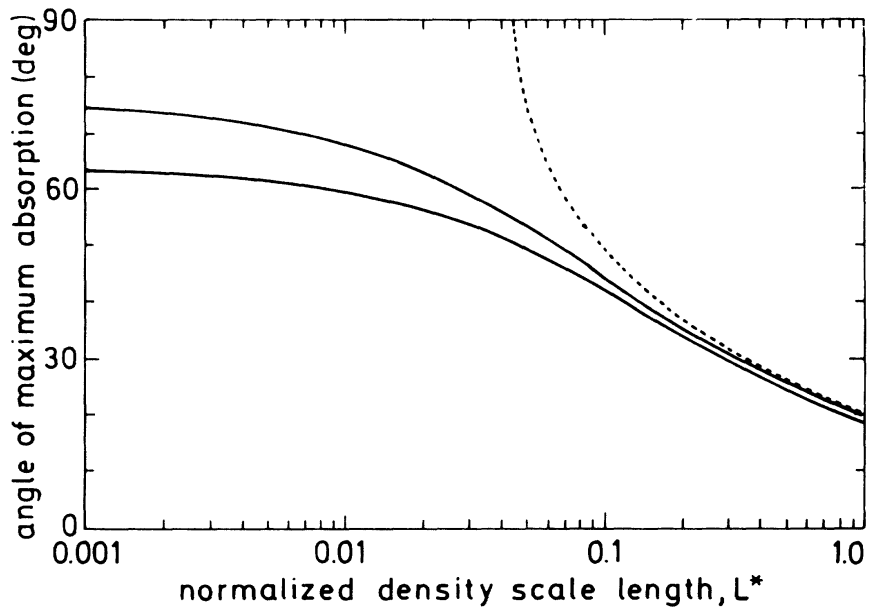

FIG. 2. Theoretical calculations of the angle of maximum absorption of $p$-polarized radiation as a function of plasma density scale length in an exponential electron-density profile: the top and bottom solid curves are for $n_{e 0}^{*}=30$ and $v_{c}^{*}=0.05$ and 0.2 , respectively, and the dashed curve is for the resonance absorption angle for long-scale-length linear density profiles.

the angle of maximum absorption for $p$-polarized light is primarily a function of the density scale length. This angle is calculated for the case of $n_{e 0}^{*}=30$ (an average value for the experimental conditions) and for normalized collision frequencies (specified now at $n_{c}$ ) of $v_{c}^{*}=0.05$ and 0.2 which are approximately the limiting values appropriate to our experimental conditions. As one would expect the numerically calculated curves connect smoothly the limiting cases of the Fresnel angle and the long-gradient-length angle.

Using the numerical results of Fig. 2 one can relate the experimental angles of maximum absorption to the plasma gradient length. One finds scale lengths of 4-20 $\mathrm{nm}$ for the measured angles of $(48-57)^{\circ}$. These numbers are in very reasonable agreement with estimates obtained by modeling the expansion as an isothermal rarefaction wave. Note that the slight shift of $\theta_{m}$ towards lower values with higher intensity seen in the experiments is attributed to the increase of the expansion velocity with intensity. However, because the sound velocity is only a weak function of the intensity the shift is not very pronounced for the parameter range of the experiments. The flattening of the density profile expected at later times as the plasma expands has been previously observed on a longer ps time scale by Maaswinkel. ${ }^{22}$

It should be emphasized that the density gradient length shows no anomaly for the high-intensity case where some plasma formation by the prepulse is expected. Thus this plasma is obviously very underdense and has little influence on the interaction of the main part of the pulse with the target.

From the numerical calculations least-square fits to the $p$-polarized data points were obtained for the four cases shown in Fig. 1. For simplicity and generality the 
numerical calculations assumed a collision frequency which was directly proportional to density $\left(v^{*} \propto n_{e}\right)$ without the indirect dependence of the Coulomb logarithm term $\left(v^{*} \propto n_{e} \ln \Lambda\right)$. The quality of the fit depends somewhat on the assumed density profile. For example, for aluminum [Figs. 1(a) and 1(c)] a linear profile provides a better fit than an exponential one. The values of $v_{c}^{*}$ corresponding to these fits are $0.13 \pm 0.02$ and $0.19 \pm 0.04$ for aluminum and $0.15 \pm 0.02$ and 0.22 \pm 0.04 for gold at the lower and higher intensities, respectively. For the aluminum cases the same best-fit values of $v_{c}^{*}$ are obtained from both the linear- and exponential-profile fits. From the observed angular dependence it is clear that the interaction occurs in an expanded plasma profile at densities at and above the critical density. For the low-intensity aluminum case the absorption occurs over a region of 1-10 times critical density, as determined by the simulations, leading to an average interaction density of $5.5 n_{c}$. The collision frequency at this effective interaction density as determined from the best-fit numerical calculation is $v^{*}=0.7 \pm 0.1$. The expected value of the classical Coulomb collision frequency at the same density and the previously estimated temperature $\left(n_{e}^{*}=5.5, T_{e}=57 \mathrm{eV}\right.$, and $\ln \Lambda$ $=1.32$ ) is $v^{*}=0.66$. This agreement appears to be quite good given the assumption of a stationary plasma profile for the present modeling. At the higher intensity the collision frequencies are found to be 2-4 times higher than expected, which is explained by the enhanced absorption in the preformed plasma as discussed earlier.

In conclusion, the present investigation reveals clearly that the reflection of intense uv femtosecond laser pulses from the dense plasma formed by irradiation of a solid has the simplicity hoped for: It can be satisfactorily explained within the framework of linear metal optics. On the other hand the onset of plasma expansion is clearly detectable and its importance will increase with longerwavelength laser light and as higher laser intensities become available. There is therefore no guarantee that this simplicity will be maintained under all conditions.

The authors would like to thank Dr. Mulser and Dr. Eidmann for useful discussions. This work was supported in part by the Bundesministerium für Forschung und Technologie and by the Commission of the European Communities in the framework of the Association EURATOM-Max-Planck-Institut für Plasmaphysik.

Note added.-After submission of this paper a new calculation ${ }^{23}$ of absorption in steep density profiles has appeared which is qualitatively in agreement with the results discussed here.

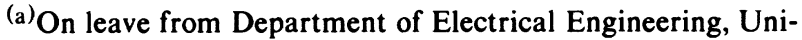
versity of Alberta, Edmonton, Canada T6G 2G7.

(b) Permanent address: Research Group on Laser Physics of the Hungarian Academy of Sciences, JATE University, Dóm tér 9, H-6720 Szeged, Hungary.

'M. Milchberg, R. R. Freeman, S. C. Davey, and R. M. More, Phys. Rev. Lett. 61, 2364 (1988).

${ }^{2}$ M. M. Murnane, H. C. Kapteyn, and R. W. Falcone, Phys. Rev. Lett. 62, 15 (1989).

${ }^{3}$ J. C. Kieffer, P. Audebert, M. Chaker, J. P. Matte, H. Pépin, T. W. Johnston, P. Maine, D. Meyerhofer, J. Delettrez, D. Strickland, P. Bado, and G. Mourou, Phys. Rev. Lett. 62, 760 (1989).

${ }^{4}$ D. Kühlke, U. Herpes, and D. von der Linde, Appl. Phys. Lett. 50, 1785 (1987).

${ }^{5}$ C. H. Nam, W. Tighe, S. Suckewer, J. F. Seely, U. Feldman, and L. A. Woltz, Phys. Rev. Lett. 59, 2427 (1987).

${ }^{6}$ D. G. Stearns, O. L. Landen, E. M. Campbell, and J. H. Scofield, Phys. Rev. 37, 1684 (1988).

${ }^{7}$ G. Kühnle, F. P. Schäfer, S. Szatmari, and G. D. Tsakiris, Appl. Phys. B 47, 361 (1988).

${ }^{8}$ J. A. Cobble, G. A. Kyrala, A. A. Hauer, A. J. Taylor, C. C. Gomez, N. D. Delamater, and G. T. Schappert, Phys. Rev. A 39, 454 (1989).

${ }^{9}$ H. W. K. Tom and O. R. Wood, II, Appl. Phys. Lett. 54, 517 (1989).

${ }^{10}$ S. E. Harris and J. D. Kmetec, Phys. Rev. Lett. 61, 62 (1988).

${ }^{11} \mathrm{P}$. Mulser, S. Pfalzner, and F. Cornolti, in Laser Interaction with Matter, edited by G. Verlade, E. Minguez, and J. M. Perlado-(World Scientific, Singapore, 1989), p. 142.

${ }^{12} \mathrm{M}$. Born and E. Wolf, Principles of Optics (Pergamon, Oxford, 1980), 6th ed.

${ }^{13}$ K. R. Manes, V. C. Rupert, J. M. Auerbach, P. Lee, and J. E. Swain, Phys. Rev. Lett. 39, 281 (1977).

${ }^{14}$ S. Szatmari and F. P. Schäfer, Opt. Commun. 68, 196 (1988).

${ }^{15}$ S. Szatmari, G. Kühnle, J. Jasny, and F. P. Schäfer, Appl. Phys. B 49, 239 (1989).

${ }^{16}$ S. Szatmari and G. Kühnle, Opt. Commun. 69, 60 (1988).

${ }^{17}$ R. Fedosejevs, R. Ottmann, R. Sigel, G. Kühnle, S. Szatmari, and F. P. Schäfer, Appl. Phys. B (to be published).

${ }^{18}$ Ya. B. Zel'dovich and Yu. P. Raizer, Physics of Shock Waves and High-Temperature Hydrodynamic Phenomena (Academic, New York, 1966).

${ }^{19}$ L. Spitzer, Jr., Physics of Fully Ionized Gases (Wiley, New York, 1962).

${ }^{20}$ R. M. More, Adv. At. Mol. Phys. 21, 305 (1985).

${ }^{21}$ V. L. Ginzburg, The Propagation of Electromagnetic Waves in Plasmas (Pergamon, Oxford, 1970), 2nd ed.

${ }^{22}$ A. G. M. Maaswinkel, Z. Naturforsch. 35a, 1162 (1980).

${ }^{23}$ H. M. Milchberg and R. R. Freeman, J. Opt. Soc. Am. B 6, 1351 (1989). 